\title{
Association of prostate volume with incidence and aggressiveness of prostate cancer
}

This article was published in the following Dove Press journal:

Research and Reports in Urology

26 October 2016

Number of times this article has been viewed

\section{Shadi Al-Khalil \\ Christine Ibilibor James Thomas Cammack Werner de Riese}

Department of Urology, Texas Tech University Health Sciences Center, Lubbock, TX, USA
Correspondence: Werner de Riese Department of Urology, Texas Tech University Health Sciences Center, 360I 4th Street, Suite 3BI00, Lubbock, TX 79430, USA

Tel +l 8067431810

Email werner.deriese@ttuhsc.edu
Objective: The aim of this study was to investigate the possible correlation between prostate volume and aggressiveness and incidence of prostate cancer ( $\mathrm{PCa})$.

Patients and methods: A chart review of a cohort of 448 consecutive prostate biopsy-naive men was performed. These men underwent at least a 12-core biopsy at our institution due to increased prostate-specific antigen serum levels $(>4 \mathrm{ng} / \mathrm{mL})$ and/or suspicious findings on digital rectal examination during the period between 2008 and 2013. Transrectal ultrasound was used to determine the prostate volume.

Results: The positive biopsy rate was $66 \%$ for patients with a prostate volume of $\leq 35 \mathrm{cc}$ and $40 \%$ for patients with a prostate volume of $\geq 65 \mathrm{cc}(P<0.001)$. Of the 110 patients testing positive on biopsy with a volume of $\leq 35 \mathrm{cc}, 10$ patients $(9.1 \%)$ had a Gleason score of $\geq 8$. Of the 27 patients testing positive on biopsy with a volume of $\geq 65 \mathrm{cc}$, only 1 patient $(3.7 \%)$ had a Gleason score of $\geq 8$.

Conclusion: These results suggest that there may be an association between prostate volume and the incidence and aggressiveness of PCa. The larger the prostate, the lower the positive biopsy rate for $\mathrm{PCa}$ and the lower the Gleason score.

Keywords: prostate cancer, prostate-specific antigen, prostate volume

\section{Introduction}

Benign prostatic hyperplasia $(\mathrm{BPH})$ and prostate cancer $(\mathrm{PCa})$ are the most common diseases of the prostate; however, their interaction is not well studied. ${ }^{1,2}$ Historically, elucidating the epidemiology of BPH has been complicated by the lack of a uniform definition of clinical BPH, quantitative instruments for assessing the severity of lower urinary tract symptoms, and a noninvasive and accurate method for measuring both prostate volume and bladder outlet obstruction. Several investigators in different countries found that the prevalence of clinical BPH is fairly uniform worldwide and also consistently age related. ${ }^{1}$ Symptomatic BPH affects $\sim 20 \%$ of men in the age-group of 50-59 years, $30 \%$ of men in the age-group of $60-69$ years, and $40 \%$ of men in the age-group of 70 years and older. ${ }^{3}$

Currently, PCa also remains a significant burden in the health of older men. PCa is the most commonly diagnosed non-skin cancer in men, accounting for $21 \%$ of newly diagnosed non-skin cancers in 2016; this amounts to 180,890 new cases of PCa, including 26,120 deaths in 2016. ${ }^{4}$

Prostate-specific antigen (PSA) is a widely used, albeit controversial, diagnostic tool in the detection of PCa. Serum PSA levels of $>4.0 \mathrm{ng} / \mathrm{mL}$ has a sensitivity of 
$\sim 20 \%$ and a specificity in the range of $60-70 \% .^{5}$ The low sensitivity may be due to the fact that PSA serum levels can be elevated in the presence of benign pathology such as BPH and prostatitis.

Recent clinical studies have shown that there may be an association between prostate volume and the incidence of $\mathrm{PCa} .^{2,6-9}$ In this context, we performed a study to further elucidate the relationship between prostate volume and the incidence of PCa.

\section{Patients and methods}

A cohort of 448 men were seen and evaluated at the Urology Clinic of Texas Tech Physicians in Lubbock, TX, USA, from 2008 to 2013. Patients who never had a biopsy with PSA levels of $>4 \mathrm{ng} / \mathrm{mL}$ or with suspicious findings on digital rectal examination (DRE) were included in our study. For each patient prior to performing a warranted prostate biopsy, a complete history was collected and physical examination was performed. The prostate volume for each patient was estimated by DRE and confirmed by transrectal ultrasound (TRUS). Patients who underwent prior biopsies or prior surgeries were excluded from our study. For each prostate, 12-core TRUS-guided biopsies were performed bilaterally, including at the apex, base, and middle portion of the gland. A minimum of six biopsies were performed in each lobe in addition to biopsies obtained if suspicious lesions were encountered. PSA density data were obtained by dividing the PSA serum level by the TRUS-confirmed prostate volume.

The Texas Tech University Health Sciences Center Institutional Review Board For The Protection Of Human Subjects approved the study (IRB: L14-107). Due to the retrospective nature of the study the review board waived the need for patient written informed consent and Health Insurance Portability and Accountability Act (HIPAA) authorization. Statistical analysis was performed by Statistical Analysis System program, Windows 9.1.3.

\section{Results}

Of the 442 patients who underwent prostate biopsy, 244 patients $(55.2 \%)$ were found to have a positive biopsy result. The rate of positive biopsy correlated with an increase in age: $48.28 \%$ in the age range of $40-54$ years inclusively, $53.87 \%$ in the age range of 55-69 years inclusively, and $61.9 \%$ in the age range of 70 years and older (Table 1). In addition, mean Gleason score, representing the aggressiveness of the diagnosed $\mathrm{PCa}$, had a similar relationship with respect to increasing age. Distribution of PSA, DRE-determined volume, and TRUS-determined volume are graphically represented in Figure 1.

Next, we determined the positive biopsy rates with respect to prostate volume. Prostates with a positive biopsy were divided into three groups based on prostate volume: prostates $<35 \mathrm{cc}$, prostates between 35 and $65 \mathrm{cc}$, and prostates $>65 \mathrm{cc}$. The results are shown in Figure 2. The positive biopsy rate was $66 \%$ for patients with a prostate volume of $\leq 35 \mathrm{cc}$ and $40 \%$ for patients with a prostate volume of $\geq 65 \mathrm{cc}(P<0.001)$.

We then determined the Gleason scores for prostates with a volume of $<35$ and $>65 \mathrm{cc}$. The results are shown in Table 2 . Of the 110 patients testing positive on biopsy with a volume of $<35$ cc, 10 patients $(9.09 \%)$ had a Gleason score of $\geq 8$. Of the 27 patients testing positive on biopsy with a volume of $>65 \mathrm{cc}, 1$ patient $(3.7 \%)$ had a Gleason score of $\geq 8$.

\section{Discussion}

Several studies have demonstrated a relationship between prostate volume and the incidence of $\mathrm{PCa} .{ }^{2,6-9}$ These studies have shown an inverse relationship between prostate volume and the incidence of $\mathrm{PCa}$; as prostate volume increases, incidence of $\mathrm{PCa}$ decreases. Our results exhibit a similar relationship as indicated in Figure 2. Prostate volume of $\leq 35 \mathrm{cc}$ had a $66 \%$ positive biopsy rate, whereas prostate volume of $\geq 65$ cc had a $40 \%$ positive biopsy rate, ie, a reduction of $39.4 \%$. A recent study described an inverse relationship between prostate symptom score and $\mathrm{PCa} .{ }^{10}$ Since a prostate

Table I Clinical parameters of patients undergoing prostate biopsies

\begin{tabular}{|c|c|c|c|c|c|}
\hline Parameters & 40-54 years & $55-69$ years & $70+$ years & Total & $P$-value \\
\hline Number of patients & 58 & 271 & 113 & 442 & \\
\hline Positive biopsies, n (\%) & $28(48.28)$ & $146(53.87)$ & $70(61.90)$ & $244(55.20)$ & $P<0.001$ \\
\hline Mean Gleason score & 6.57 & 6.71 & 6.86 & 6.74 & $P<0.025$ \\
\hline Abnormal DRE & $60 \%$ & $64.40 \%$ & $69.52 \%$ & $65.76 \%$ & \\
\hline Mean DRE-estimated volume (mL) & 32.04 & 36.99 & 41.51 & 37.46 & $P<0.05$ \\
\hline Mean TRUS-determined volume $(\mathrm{mL})$ & 31.32 & 42.89 & 52.03 & 43.62 & $P<0.05$ \\
\hline Mean PSA & 8.91 & 14.05 & 19.45 & 11.78 & $P<0.05$ \\
\hline Median PSA & 5.4 & 6.2 & 7.3 & 6.3 & \\
\hline Patients with a family history & $28 \%$ & $17.09 \%$ & $16.13 \%$ & $19.67 \%$ & \\
\hline
\end{tabular}

Note: ${ }^{a}$ Abnormal DRE is defined as increased size of $>30$ g, nodules, or induration.

Abbreviations: DRE, digital rectal examination; PSA, prostate-specific antigen; TRUS, transrectal ultrasound. 
A

PSA vs age

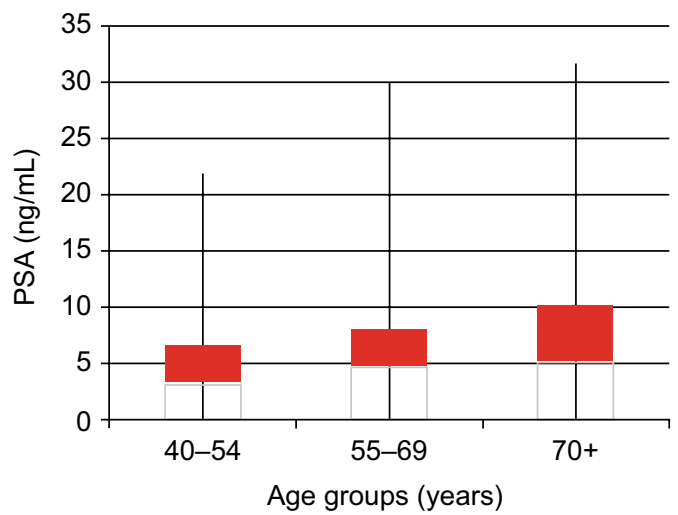

B

DRE volume vs age

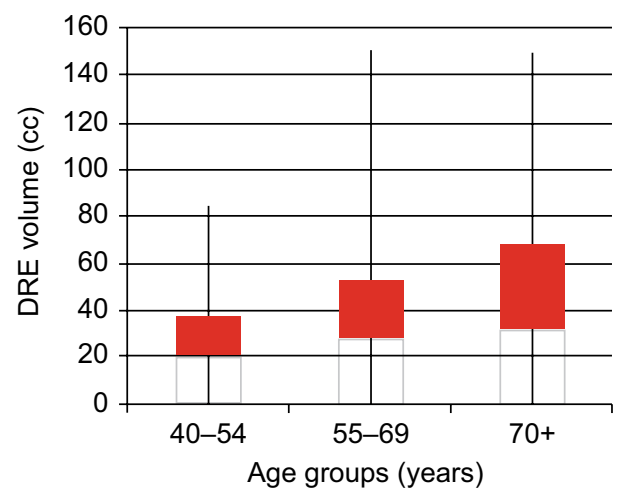

C

TRUS volume vs age

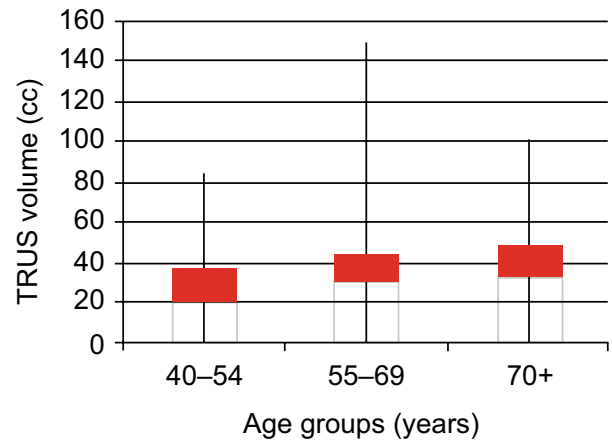

Figure I Distribution of PSA (A) and DRE- (B) and TRUS-determined (C) volumes among age groups.

Note: Cases with subsequently proven metastatic disease were excluded.

Abbreviations: DRE, digital rectal examination; PSA, prostate-specific antigen; TRUS, transrectal ultrasound.

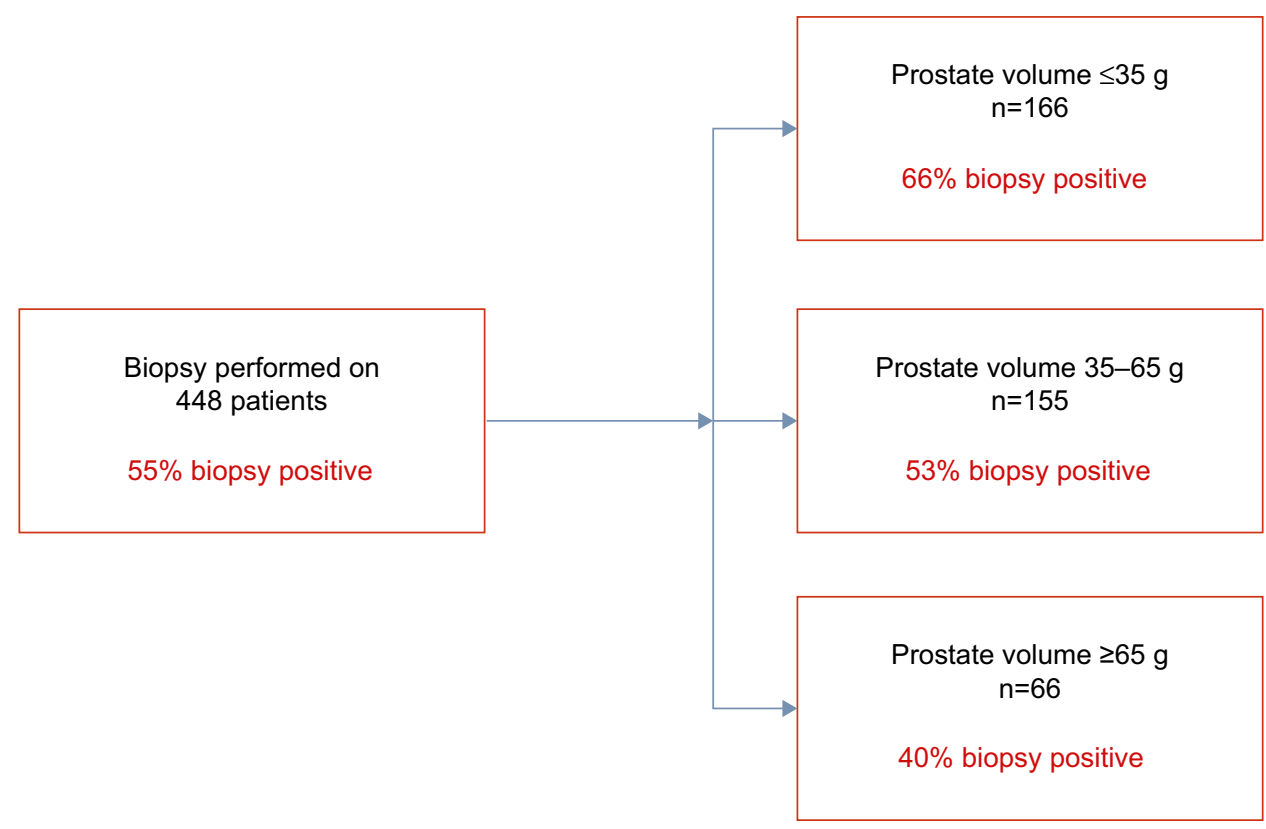

Figure 2 Categorical breakdown by prostate volume.

Abbreviation: TRUS, transrectal ultrasound. 
Table 2 Gleason scores by prostate volume

\begin{tabular}{llll}
\hline Parameters & $\begin{array}{l}\text { Volume } \\
<35 \text { cc }\end{array}$ & $\begin{array}{l}\text { Volume } \\
>65 \text { cc }\end{array}$ & P-value \\
\hline Number of patients & 166 & 66 & \\
Biopsy-positive patients, n (\%) & $110(66.27)$ & $27(40.9)$ & $P<0.00 I$ \\
Patients with Gleason score $\leq 7, \mathrm{n}(\%)$ & $100(90.9)$ & $26(96.29)$ & $P<0.03$ \\
Patients with Gleason score $\geq 8, \mathrm{n} \mathrm{( \% )}$ & $10(9.09)$ & $\mathrm{I}(3.7)$ & \\
\hline
\end{tabular}

symptom score would correlate with prostate volume, this pattern would be expected when compared with the studies mentioned earlier., ${ }^{2,-9}$ However, this study could not show an increase in the accuracy of cancer detection in the multivariate analysis..$^{10}$ Another study has shown an association between prostate volume and high-grade advanced $\mathrm{PCa} .{ }^{11}$ A recent publication examined the association of $\mathrm{PCa}$ volume and prostate size. Their data showed that small-volume cancers $(<0.5 \mathrm{~g})$ were twice as common in larger glands ( $>50 \mathrm{~g}$ ) compared to smaller glands $(<50 \mathrm{~g})$. This may imply that PCa may spread with less difficulty in smaller glands. ${ }^{9,11}$ In another recent prospective study, a large cohort of 1,044 men underwent multiparametric magnetic resonance imaging (MRI) and then 12-core systemic mapping biopsy with additional MRI-fusion ultrasound (US) biopsy if the previous MRI had detected suspicious lesions of the prostate. This large study also revealed an inverse association of prostate volume with incidence and higher Gleason score (>7) for PCa. ${ }^{12}$ The above-mentioned studies, in addition to the results of our study, support the hypothesis of an inverse association between prostate volume and the incidence of PCa. Furthermore, the results in Table 2 illustrate a reduction in high-grade $\mathrm{PCa}$ (Gleason score of $\geq 8$ ) in larger prostates (volume $>65 \mathrm{cc}$ ). Out of 110 biopsy-positive patients with prostates of $<35 \mathrm{cc}, 10$ patients had a Gleason score of $\geq 8$ (9.1\%). However, out of 27 biopsy-positive patients with prostates of $>65 \mathrm{cc}$, only 1 patient had a Gleason score of $\geq 8$ (3.7\%). This is a reduction of $59.3 \%$ in high-grade cancers when comparing prostates to a volume of $<35$ and $>65 \mathrm{cc}$. Therefore, our results show that not only the incidence but also the aggressiveness of $\mathrm{PCa}$ decreases in larger prostates.

Other previous clinical studies have also described the phenomenon of decreased cancer detection rates in larger prostates. ${ }^{11,13-15}$ The authors debated that increased prostate volume makes it more difficult to detect same-size cancer lesions with the biopsy needle compared to smaller prostates with the same cancer volume lesions. These authors labeled this issue of lower PCa detection rates in enlarged prostates as the so-called sampling error. ${ }^{13}$ Recently, TRUS-guided biopsy was believed to be the limiting factor in the detection of PCa in larger prostates as 12-core TRUS biopsies may under-sample large glands. Thus, prostate biopsy protocols have evolved during the recent years toward protocols that propose increasing the number of biopsies. Several studies have shown PCa detection rates of $\sim 40 \%$ or less in men with a prostate volume of $>50 \mathrm{cc}$, while, and in contrast, detection rates were much higher in smaller glands. ${ }^{16,17}$ In the past few years, the MRI-TRUS fusion imaging with targeted biopsies has evolved overcoming the limitations of the TRUS-guided biopsy technique, in particular, for larger prostates. De Gorski et $\mathrm{al}^{7}$ describe the improved detection rate for this malignancy in larger prostates compared to US-guided biopsies alone. In this study, the detection rate for MRI-TRUS-fusion-guided biopsy was $77 \%$ in prostate glands with a volume of $<30 \mathrm{cc}$, compared to $61 \%, 47 \%$, and $34 \%$ for glands with a volume of 30 to $<38.5 \mathrm{cc}, 38.5$ to $<55 \mathrm{cc}$, and $>55 \mathrm{cc}$, respectively. ${ }^{17}$ These results were statistically significant $(P=0.001)$. These data are similar and correspond well with the results described in our study $(66.27 \%$ in prostates $<35 \mathrm{cc}$ and $40.9 \%$ in glands $>65 \mathrm{cc}$ ). Thus, the finding that MRI-TRUS fusion imaging improved the detection of suspicious prostate lesions and was not impaired by prostate size endorses the assumption of inverse correlation between prostate volume and cancer incidence. Therefore, the argument that this observed association between prostate volume and the incidence of cancer is due to sampling error may be contested. ${ }^{12}$

Studies have shown that $80 \%$ of $\mathrm{PCa}$ arises in the peripheral zone (PZ), whereas BPH is caused by growth of the transition zone (TZ). ${ }^{18}$ To the experienced urologists, it is a well-known phenomenon in open surgical treatment of large BPH glands with a size of $>80-90 \mathrm{cc}$ that the continuous growth of the $\mathrm{TZ}$ compresses the $\mathrm{PZ}$ and thus creates the so-called surgical capsule of the prostate, which enables enucleating the BPH component and leaving the collagenrich and cell-deprived surgical capsule behind. ${ }^{19}$ The clinical question arises whether the BPH-related TZ enlargement could cause enough atrophy, scarring, and apoptosis of the epithelial cells in the PZ and thus significantly reduce the risk of developing adenocarcinoma of the prostate in the remaining epithelial glands. This might explain the reduced detection rate of $\mathrm{PCa}$ in large prostates as outlined earlier.

\section{Limitations}

We report in this study a retrospective data review, which has several limitations including its single-center design, the lack of rationale for prostate volume ranges, the limited sample size of larger prostate volume, and the uneven distribution of cases according to the selected prostate volume ranges. Furthermore, without evaluating the whole-mount prostatectomy specimens for those patients who underwent surgery, the difference in 
accuracy between prostate biopsies and the final pathology findings of the entire gland specimen could not be determined.

\section{Conclusion}

The results of this study depict an inverse association of prostate volume with the incidence and biological aggressiveness of PCa. Data from this study and the outlined discussion should encourage other clinicians and investigators to further explore the relationship between prostate volume and the incidence and aggressiveness of $\mathrm{PCa}$, to further investigate this phenomenon. This, in turn, may have future clinical implications.

\section{Acknowledgment}

We would like to acknowledge the Clinical Research Office at Texas Tech University Health Sciences Center in supporting the IRB application.

\section{Disclosure}

The authors report no conflicts of interest in this work.

\section{References}

1. Lepor H. Pathophysiology, epidemiology, and natural history of benign prostatic hyperplasia. Rev Urol. 2004;6(suppl 9):S3-S10.

2. Al-Khalil S, Boothe D, Durdin T, et al. Possible interactions between benign prostatic hyperplasia $(\mathrm{BPH})$ and prostate cancer in large prostates. Int Urol Nephrol. 2016;48(1):91-97.

3. Bosch JL, Hop WC, Kirkels WJ, Schröder FH. The international prostate symptom score in a community-based sample of men between 55 and 74 years of age: prevalence and correlation of symptoms with age, prostate volume, flow rate, and residual urine volume. $\mathrm{Br} J \mathrm{Urol}$. 1995;75(5):622-630.

4. Siegel RL, Miller KD, Jemal A. Cancer statistics, 2016. CA Cancer J Clin. 2016;66(1):7-30.

5. Gosselaar C, Roobol MJ, Roemeling S, et al; European Randomized Study of Screening for Prostate Cancer (ERSPC). Screening for prostate cancer without digital rectal examination and transrectal ultrasound: results after four years in the European Randomized Study of Screening for Prostate Cancer (ERSPC), Rotterdam. Prostate. 2006;66(6):625-631.
6. Kim JS, Ryu JG, Kim JW, et al. Prostate-specific antigen fluctuation: what does it mean in diagnosis of prostate cancer? Int Braz J Urol. 2015;41(2): 258-264.

7. De Gorski A, Roupret M, Peyronnet B, et al. Accuracy of magnetic resonance imaging/ultrasound fusion targeted biopsies to diagnose clinically significant prostate cancer in enlarged compared to smaller prostates. J Urol. 2015;194(3):669-673.

8. Levine MA, Ittman M, Melamed J, Lepor H. Two consecutive sets of transrectal ultrasound guided sextant biopsies of the prostate for the detection of prostate cancer. J Urol. 1998;159(2):471-476.

9. Chen ME, Troncoso P, Johnston D, et al. Prostate cancer detection: relationship to prostate size. Urology. 1999;53(4):764-768.

10. Oh JJ, Jeong SJ, Jeong CW, et al. Is there any association between the severity of lower urinary tract symptoms and the risk of biopsydetectable prostate cancer in patients with PSA level below $20 \mathrm{ng} / \mathrm{ml}$ in multi-core prostate biopsy? Prostate. 2013;73(1):42-47.

11. Freedland SJ, Isaacs WB, Platz EA, et al. Prostate size and risk of high-grade, advanced prostate cancer and biochemical progression after radical prostatectomy: a search database study. J Clin Oncol. 2005;23(30):7546-7554.

12. Filson C, Margolis D, Huang J, et al. MR-US fusion biopsy: importance of both systematic and targeted sampling to diagnose prostate cancer. American Urological Association Annual Meeting; May 15, 2015; New Orleans, LA.

13. Ung JO, San Francisco IF, Regan MM, DeWolf WC, Olumi AF. The relationship of prostate gland volume to extended needle biopsy on prostate cancer detection. J Urol. 2003;169(1):130-135.

14. Kozminski MA, Palapattu GS, Mehra R, et al. Understanding the relationship between tumor size, gland size, and disease aggressiveness in men with prostate cancer. Urology. 2014;84(2):373-378.

15. Hong SK, Poon BY, Sjoberg DD, et al. Prostate size and adverse pathologic features in men undergoing radical prostatectomy. Urology. 2014;84(1):153-157.

16. Werahera PN, Sullivan K, La Rosa FG, et al. Optimization of prostate cancer diagnosis by increasing the number of core biopsies based on gland volume. Int J Clin Exp Pathol. 2012;5(9):892-899.

17. Yoon BI, Shin TS, Cho HJ, et al. Is it effective to perform two more prostate biopsies according to prostate-specific antigen level and prostate volume in detecting prostate cancer? Prospective study of 10-core and 12-core prostate biopsy. Urol J. 2012;9(2):491-497.

18. Marks LS, Roehrborn CG, Wolford E, WilsonTH. The effect of dutasteride on the peripheral and transition zones of the prostate and the value of the transition zone index in predicting treatment response. JUrol. 2007;177(4): 1408-1413.

19. Ayala AG, Ro JY, Babaian R, Troncoso P, Grignon DJ. The prostatic capsule: does it exist? Its importance in the staging and treatment of prostatic carcinoma. Am J Surg Pathol. 1989;13(1):21-27.
Research and Reports in Urology

\section{Publish your work in this journal}

Research and Reports in Urology is an international, peer-reviewed, open access journal publishing original research, reports, editorials, reviews and commentaries on all aspects of adult and pediatric urology in the clinic and laboratory including the following topics: Pathology, pathophysiology of urological disease; Investigation and treatment of
Dovepress

urological disease; Pharmacology of drugs used for the treatment of urological disease. The manuscript management system is completely online and includes a very quick and fair peer-review system, which is all easy to use. Visit http://www.dovepress.com/testimonials.php to read real quotes from published authors. 